\title{
71
}

\section{A Critical Analysis of the DESSERT Information Model}

\author{
Richard MEADE, Ahmed PATEL \\ University College Dublin, IRELAND \\ Declan O'SULLIVAN, Mark TIERNEY \\ Broadcom Eirann Research, IRELAND
}

This poster looks at a number of different Information Models which have been developed within various problem domains related to network management and highlights the important similarities. Furthermore it considers one particular problem domain, service provisioning, and an Information Model which was developed for it, in which the benefits of Information Modelling are particularly apparent because of the wide scope and characteristics of the domain.

Finally, we also propose a new approach to modelling networks traffic, based on this model. This will enable modelling of high level and low level details while providing a more flexible and more complete method of modelling characteristics such as network connectivity and topology. It also enables easier and more appropriate modelling of quality of service parameters. 